\title{
DNAJB6 is a peptide-binding chaperone which can suppress amyloid fibrillation of polyglutamine peptides at substoichiometric molar ratios
}

\author{
Cecilia Månsson • Vaishali Kakkar • Elodie Monsellier • \\ Yannick Sourigues • Johan Härmark • Harm H. Kampinga • \\ Ronald Melki • Cecilia Emanuelsson
}

Received: 5 June 2013 /Revised: 10 July 2013 / Accepted: 11 July 2013 / Published online: 1 August 2013

(C) The Author(s) 2013. This article is published with open access at Springerlink.com

\begin{abstract}
Expanded polyglutamine (polyQ) stretches lead to protein aggregation and severe neurodegenerative diseases. A highly efficient suppressor of polyQ aggregation was identified, the DNAJB6, when molecular chaperones from the HSPH, HSPA, and DNAJ families were screened for huntingtin exon 1 aggregation in cells (Hageman et al. in Mol Cell 37(3):355-369, 2010). Furthermore, also aggregation of polyQ peptides expressed in cells was recently found to be efficiently suppressed by co-expression of DNAJB6 (Gillis et al. in J Biol Chem 288:17225-17237, 2013). These suppression effects can be due to an indirect effect of DNAJB6 on other cellular components or to a direct interaction between DNAJB6 and polyQ peptides that may depend on other cellular components. Here, we have purified the DNAJB6 protein to investigate the suppression mechanism. The purified DNAJB6 protein formed large heterogeneous oligomers, in contrast to the more canonical family member DNAJB1 which is dimeric. Purified DNAJB6 protein, at substoichiometric
\end{abstract}

Electronic supplementary material The online version of this article (doi:10.1007/s12192-013-0448-5) contains supplementary material, which is available to authorized users.

C. Månsson $(\bowtie) \cdot$ C. Emanuelsson

Department of Biochemistry \& Structural Biology, Center for

Molecular Protein Science, Lund University, Lund, Sweden

e-mail: cecilia.mansson@biochemistry.lu.se

V. Kakkar $\cdot$ H. H. Kampinga

Department of Cell Biology, UMCG, Groningen, The Netherlands

E. Monsellier $\cdot$ Y. Sourigues $\cdot$ R. Melki

Laboratoire d'Enzymologie et Biochimie Structurales, Centre

National de la Recherche Scientifique, Gif-sur-Yvette, Paris, France

J. Härmark

Department of Biosciences and Nutrition, Karolinska Institute,

Huddinge, Sweden molar ratios, efficiently suppressed fibrillation of polyQ peptides with $45^{\circ} \mathrm{Q}$ in a thioflavin $\mathrm{T}$ fibrillation. No suppression was obtained with DNAJB1, but with the closest homologue to DNAJB6, DNAJB8. The suppression effect was independent of HSPA1 and ATP. These data, based on purified proteins and controlled fibrillation in vitro, strongly suggest that the fibrillation suppression is due to a direct protein-protein interaction between the polyQ peptides and DNAJB6 and that the DNAJB6 has unique fibrillation suppression properties lacking in DNAJB1. Together, the data obtained in cells and in vitro support the view that DNAJB6 is a peptide-binding chaperone that can interact with polyQ peptides that are incompletely degraded by and released from the proteasome.

Keywords Aggregation · Amyloid · DNAJ · Molecular chaperones $\cdot$ Polyglutamine

\section{Introduction}

Among the various molecular chaperone families, the DNAJ family is the largest and most heterogeneous group, named after their common nominator, the $\mathrm{J}$ domain, which is responsible for the interactions with the ATP-dependent Hsp70 (HSPA) refolding machinery. Canonically, the DNAJ proteins bind to substrate proteins, transport, and present them to Hsp70 for refolding. The J domain also stimulates the ATPase activity of Hsp70. The DNAJ family members are thought to be responsible for substrate specificity (Kampinga and Craig 2010). Molecular chaperones in the protein quality control system assist folding and degradation of substrate proteins (Tyedmers et al. 2010) and suppress aggregation and cytotoxicity induced by heat shock or other proteotoxic insults (Kampinga 1993; Morimoto 2008). 
Neurodegenerative polyglutamine diseases are caused by a CAG triplet expansion in the affected gene, which leads to an aggregation-prone prolonged polyglutamine (polyQ) stretch in the encoded protein (Gatchel and Zoghbi 2005). Stretches with polyQ occur in many human proteins, e.g., in transcription factors. This suggests that such polyQ stretches have an evolutionary early and important cellular function (Ramazzotti et al. 2012; Schaefer et al. 2012). The normal form of the human huntingtin $(\mathrm{Htt})$ protein contains a polyQ stretch of 11 to 34 glutamines in exon 1 (HttEx1). An expansion of the number of glutamines to above 35 leads to Huntington's disease (HD), a severe disease hallmarked by protein fibrillation and neuronal inclusion body formation, linked to a progressive neurodegenerative process with uncontrolled movements, personality changes, and cognitive impairment. The length of the polyQ stretch is statistically related to the age of onset of HD: the longer, the earlier the onset of symptoms (Novak and Tabrizi 2010). Fibrillation propensity is also dependent on polyQ length as shown in several cell and animal models as well as in vitro data with purified proteins (de Almeida et al. 2002; Kar et al. 2011). All these suggest that aggregation initiates disease, although the nature of the toxic entities (fibrils or precursor oligomeric forms) that cause neurodegeneration is subject to debate.

Various molecular chaperones have been studied for their involvement in polyQ diseases (Gestwicki and Garza 2012). Within the DNAJ protein family, the HSJ1a (DNAJB2a) (Labbadia et al. 2012) and DNAJB1 (Lotz et al. 2010) have been reported to suppress polyQ aggregation in cooperation with the Hsp70 machinery. However, in a cell-based screen of various molecular chaperones from the HSPH, HSPA, and DNAJ families, the most effective to suppress polyQ aggregation are two closely related DNAJ homologues, DNAJB6 and DNAJB8 (Hageman et al. 2010). Suppression had previously been observed for DNAJB6 in a human cell line (Chuang et al. 2002) and for the Drosophila ortholog Mrj1 (Fayazi et al. 2006). Interestingly, the suppression of polyQ aggregation by DNAJB6 (Hageman et al. 2010) was found to be independent of the Hsp70 machinery. In fact, the Nterminal J domain, required for Hsp70 interactions, was largely dispensable for this effect.

The mechanism of polyQ fibrillation and the influence of the polyQ context within proteins are not fully understood at the molecular level (Bieschke et al. 2012; Wetzel 2012). Aggregation in nine different polyQ diseases (HD, x-linked spinal and bulbar muscular atrophy, dentatorubralpallidoluysian atrophy and six forms of spinocerebellar ataxia) is tightly associated with polyQ stretches in nine functionally and structurally unrelated proteins (Zoghbi and Orr 2000). Furthermore, polyQ stretches fused to green fluorescent protein (GFP) affect, for example, motility in Caenohabditis elegans models (Morley et al. 2002), and polyQ stretches alone cause toxicity in Drosophila (Marsh et al. 2000).
Thus, it appears that it is the polyQ stretches that drive aggregation.

Several reports suggest that aggregation initiation is associated with processing of polyQ-containing proteins by proteases, since the full-length polyQ-containing proteins are less prone to aggregation (Harris et al. 2010; Ratovitski et al. 2009). The context around the polyQ stretch does seem, however, important, probably, both for its processing into such smaller fragments (Wellington et al. 1998; Berke et al. 2004; Jung et al. 2009; Graham et al. 2006) as well as for the (co)aggregation proneness of the generated fragments (Dehay and Bertolotti 2006). The proteasome is unable to cleave expanded polyQ stretches (Holmberg et al. 2004; Venkatraman et al. 2004). Proteasomal processing of polyQ-containing proteins or fragments generated thereof may therefore yield highly aggregation-prone polyQ peptides (Raspe et al. 2009), not readily digested by peptidases (Menzies et al. 2010). These polyQ peptides could thus be a major initiator of aggregation and disease, by seeding the aggregation of polyQ-containing proteins. Recently, cellular polyQ aggregation, initiated by overexpression of peptides with expanded polyQ stretches, was found to be efficiently suppressed by co-expression of DNAJB6 and DNAJB8 (Gillis et al. 2013). Such suppression could be due to either an indirect effect on other cellular components or to a direct interaction between DNAJB6/DNAJB8 and polyQ peptides.

In order to obtain further evidence for a direct interaction between DNAJB6 and polyQ and to gain insight into the suppression mechanism, we decided to purify the proteins, focusing on the functional characterization of DNAJB6 since it is ubiquitously expressed, including in the brain, whereas DNAJB8 is only expressed in the testis (Hageman et al. 2010). We decided to evaluate the suppression by thioflavin $\mathrm{T}$ binding, which is among the most widely used tools in the study of amyloid fibrils (Biancalana and Koide 2010; Wu et al. 2010), using purified polyQ peptides as well as purified polyQ constructs within a HttEx 1 context. We found that the purified DNAJB6 protein formed large heterogeneous oligomers, consistent with the oligomeric state found in human cell lines, and that DNAJB6 efficiently suppressed the fibrillation of polyQ peptides under conditions where DNAJB1 and the Hsp70 machinery had no effect.

\section{Materials and methods}

Recombinant expression and purification of DNAJB6/8

The human DNAJB6b and DNAJB8, cloned and sequenced as previously described (Hageman et al. 2010), were subcloned into pET/TOPO-151 vector (Invitrogen, Paisley, UK) containing a hexa His-tag, a V5-tag, and a tobacco etch virus (TEV) protease cleavage site. The plasmids were 
transformed into Escherichia coli strain ER2566 and grown in LB medium with $50 \mathrm{mg} / \mathrm{l}$ ampicillin. Overnight cultures of $5 \mathrm{ml}$ were used to inoculate $500 \mathrm{ml}$ cultures in 2.5-1 baffled flasks in a shaker at $37{ }^{\circ} \mathrm{C}$. Isopropyl $\beta$-D-1-thiogalactopyranoside was added at $\mathrm{OD}_{600}=1$, and cells were collected 3$4 \mathrm{~h}$ later by centrifugation at $6,000 \times \mathrm{g}$ for $5 \mathrm{~min}$ and frozen at $-20{ }^{\circ} \mathrm{C}$. Cell pellets were dissolved in buffer $(20 \mathrm{mM}$ sodium phosphate buffer, $\mathrm{pH} 8.0,150 \mathrm{mM} \mathrm{NaCl}, 5 \mathrm{mM}$ dithiothreitol (DTT)), with DNAse (Sigma-Aldrich, Stockholm, Sweden) and protease inhibitor (cOmplete EDTA free; Roche Diagnostics Scandinavia AB, Stockholm, Sweden) added just prior to cell lysis, which was performed by passing twice through a French press, followed by centrifugation at $20,000 \times g$. The supernatant was discarded, the pellet was washed in the same buffer including $0.75 \%$ Tween-20, centrifugation was repeated, and pellet was finally resuspended in the same buffer without Tween-20, but with $8 \mathrm{M}$ urea, which was, thereafter, aliquoted and stored at $-20{ }^{\circ} \mathrm{C}$. For affinity chromatography, the sample was thawed and diluted tenfold in the same buffer with no urea, and imidazole was added to a final concentration of $20 \mathrm{mM}$. The sample was loaded on the HisTrap column (GE Healthcare Lifesciences, Uppsala, Sweden), washed with buffer containing $20 \mathrm{mM}$ imidazole, and eluted with buffer containing $350 \mathrm{mM}$ imidazole. The His-tag was cleaved with TEV protease (Invitrogen, Paisley, UK) at a protein concentration of $1 \mathrm{mg} / \mathrm{ml}$ and 1 unit protease per $3 \mu \mathrm{g}$ protein at $20{ }^{\circ} \mathrm{C}$ overnight. After cleavage, the sample was diluted with imidazole-free buffer and concentrated using the Centriprep centrifugal filter devices (Millipore AB Sweden, Solna, Sweden) and applied a second time to the HisTrap column. The flow-through was collected and contained the His-tag-free proteins, whereas the cleaved off His-tag, any remaining uncleaved His-tagged protein, and the TEV protease, which contains a non-cleavable His-tag, were retained on the column. Protein concentration was determined using the Bradford assay (Bradford 1976; Stoscheck 1990) with bovine serum albumin (BSA) as standard. Non-denaturing (native polyacrylamide gel electrophoresis (PAGE)) and sodium dodecyl sulfate (SDS)-PAGE was performed using precast 3-12\% NativePAGE Bis-Tris Gels and precast 4-12\% NuPAGE ${ }^{\circledR}$ SDS-PAGE Bis-Tris Gels (Life Technologies Europe BV, Stockholm, Sweden), stained with colloidal Coomassie Brilliant Blue (Lawrence and Besir 2009) and scanned with the Image Scanner III using the software Labscan/IQTL (GE Healthcare Lifesciences, Uppsala, Sweden).

\section{Dynamic light scattering}

Dynamic light scattering (DLS) measurement was used to assess particle size using the Zetasizer Nano S (Malvern
Instruments, Malvern, UK) amenable for measurements on particles between $0.3 \mathrm{~nm}$ to $10 \mu \mathrm{m}$.

\section{Size exclusion chromatography}

Size exclusion chromatography (SEC) was performed by gel filtration using the HiPrep Sephacryl S-400 16/60 column (GE Healthcare Lifesciences, Uppsala, Sweden). The column was equilibrated with buffer ( $20 \mathrm{mM}$ sodium phosphate buffer, $\mathrm{pH}$ $8.0,150 \mathrm{mM} \mathrm{NaCl}$ ) and operated at a flow rate of $0.5 \mathrm{ml} / \mathrm{min}$ at $4{ }^{\circ} \mathrm{C}$. Fractions of $0.5 \mathrm{ml}$ were collected and protein elution followed by detection of the absorbance at $280 \mathrm{~nm}$.

Expression and purification of maltose-binding protein-fused HttEx1 and polyQ peptide

Recombinant C-terminally hexa His-tagged maltose-binding protein (MBP)-TEV-HttEx1Qn and N-terminally hexa Histagged MBP-TEV-Qn of varying lengths ( $n=15$ and 45$)$ were expressed in E. coli strain BL21(DE3) (Stratagene, Santa Clara, CA, USA) and purified in three steps. The proteins were first eluted from the $10-\mathrm{ml}$ bed volume Talon metal affinity resin column (Clontech, Saint-Germain-en-Laye, France), equilibrated in $20 \mathrm{mM}$ Tris- $\mathrm{HCl}, \mathrm{pH} 7.5 ; 100 \mathrm{mM}$ $\mathrm{KCl}$; $20 \mathrm{mM}$ imidazole; $10 \%$ glycerol; and $1 \mathrm{mM}$ DTT by developing an imidazole gradient $(20-500 \mathrm{mM})$. The Histagged MBP-TEV-HttEx1Qn and the MBP-TEV-Qn of varying lengths were eluted from this column in $200 \mathrm{mM}$ imidazole. The fractions containing His-tagged MBP-TEVHttEx1Qn were pooled and loaded on the UnoQ6 column (BioRad, Marnes-la-Coquette, France) equilibrated in $20 \mathrm{mM}$ Tris-HCl, $\mathrm{pH}$ 7.5; $20 \mathrm{mM} \mathrm{KCl}$; and $1 \mathrm{mM}$ DTT. The column was washed with five bed volumes of the equilibration buffer, and the fusion proteins were eluted using a $\mathrm{KCl}$ gradient $(20-500 \mathrm{mM})$. His-tagged MBP-TEVHttEx1Qn eluted from this column at $200 \mathrm{mM} \mathrm{KCl}$, and their concentrations were determined from their absorbance at $280 \mathrm{~nm}$ using an extinction coefficient of $67840 \mathrm{M}^{-1} \mathrm{~cm}^{-1}$.

\section{Removal of MBP from HttEx1}

MBP-TEV protease, produced using the plasmid pRK1043 (Addgene, Cambridge, MA, USA), was added to the eluted protein at a $1: 5 w / w$ ratio and $100 \%$ cleavage, as assessed using SDS-PAGE, and was achieved upon incubating the mixture for $1 \mathrm{~h}$ at $37^{\circ} \mathrm{C}$. The mixture was then loaded on the $1-\mathrm{ml}$ bed volume Talon column, the column was washed with 20 bed volumes of $20 \mathrm{mM}$ Tris- $\mathrm{HCl}, \mathrm{pH} 7.5$, and $100 \mathrm{mM} \mathrm{KCl}$, and HttEx1Qn was eluted in $20 \mathrm{mM}$ Tris$\mathrm{HCl}, \mathrm{pH} 7.5 ; 100 \mathrm{mM} \mathrm{KCl}$; and $200 \mathrm{mM}$ imidazole. The protein was immediately desalted using PD-10 desalting columns (GE Healthcare, Uppsala, Sweden) equilibrated in $50 \mathrm{mM}$ HEPES-KOH, pH 7.5, and $150 \mathrm{mM} \mathrm{NaCl}$; 
aliquoted; flash frozen in liquid nitrogen; and stored at $-80{ }^{\circ} \mathrm{C}$ until use. The protein concentrations in the final eluate were systematically comparable to that following cleavage as assessed by SDS-PAGE analysis.

\section{Removal of MBP from polyQ peptide}

The fractions containing hexa His-tagged MBP-TEV-Qn $(1.5 \mathrm{mg} / \mathrm{ml})$ were incubated with His-tagged TEV $(0.3 \mathrm{mg} /$ $\mathrm{ml}$ ) (Invitrogen, Paisley, UK) for $20 \mathrm{~min}$ at room temperature. The mixture was then diluted tenfold and loaded on the 5-ml bed volume HiTrap Q FF column (GE Healthcare, Uppsala, Sweden). The column was washed with ten bed volumes of $20 \mathrm{mM}$ HEPES, $\mathrm{pH} 7.5$, and Qn peptides was eluted in $20 \mathrm{mM}$ HEPES, pH 7.5, and $300 \mathrm{mM} \mathrm{NaCl}$. Histagged MBP and TEV were trapped in-line on the 10-ml Talon column. The Qn peptides were immediately aliquoted, flash frozen in liquid nitrogen, and stored at $-80^{\circ} \mathrm{C}$ until use.

\section{Electron microscopy}

The DNAJB6 protein was applied to glow discharged carboncoated grids and stained with $1 \%$ uranyl acetate for negative stain electron microscopy (EM). Images were recorded, using a JEOL JEM2100F microscope at $200 \mathrm{kV}$ and a magnification of 40,000 times, equipped with the Tietz $4 \mathrm{k} \times 4 \mathrm{k}$ CCD camera (Tietz Video and Image Processing Systems GmbH, Gauting, Germany). HttEx1Qn and Qn assemblies were observed by TEM in the JEOL 1400 transmission electron microscope following adsorption onto carbon-coated 200-mesh grids and negative staining with $1 \%$ uranyl acetate. The images were recorded with the Gatan Orius CCD camera (Gatan, München, Germany).

Fibrillation assay based on thioflavin $\mathrm{T}$ fluorescence increase

The constructs of purified His-tagged MBP-TEV-HttEx1Qn and MBP-TEV-Qn of varying lengths were used to monitor fibril formation by continuous measurement of thioflavin $\mathrm{T}$ (ThT) fluorescence increase (Linse et al. 2007). The final volume was $100 \mu \mathrm{l}$ per well in a 96-well, low-binding, halfarea microplate (Corning 3881; Corning Incorporated Life Sciences, Acton, MA) kept on ice, while constructs from $30 \mu \mathrm{M}$ stock solutions were mixed to a final concentration of $15 \mu \mathrm{M}$ (or as else stated) with filtered and degassed buffer (Tris- $\mathrm{HCl}, \mathrm{pH} 7.4$ and $150 \mathrm{mM} \mathrm{KCl}$ ); the final concentration of ThT was $10 \mu \mathrm{M}$, and the final concentration of DNAJB6, DNAJB8, and DNAJB1 (obtained from Sigma-Aldrich, Stockholm, Sweden) was varied as stated in figure legends. All molar ratios between proteins are calculated on a monomer-to-monomer basis, although the same oligomeric DNAJB6 protein was used in all assays. HSPA1 (SigmaAldrich, Stockholm, Sweden) was added in a molar ration to DNAJB of 2:1, together with $2 \mathrm{mM}$ ATP and $5 \mathrm{mM} \mathrm{MgCl}_{2}$.
After adding TEV protease (molar ratio protein to protease 50:1), the microplate plate was sealed with a plastic film to avoid evaporation and immediately inserted into the plate reader FLUOstar Omega (BMG Labtech, Offenburg, Germany) and incubated at $37{ }^{\circ} \mathrm{C}$ without agitation. ThT fluorescence was recorded every $150 \mathrm{~s}$ through the bottom of the plate with excitation and emission filters of 440 and $480 \mathrm{~nm}$, respectively. Fibrillation under seeded conditions was performed by adding preformed fibrils, from a fibrillation assay experiment on the previous day, corresponding to an additional 5 or $20 \%$ of the monomer concentration $(10 \mu \mathrm{M})$.

Fibrillar assembly monitored as formation of SDS-insoluble material

The assembly of purified HttEx1Qn and Qn peptides into fibrils was monitored also by SDS-PAGE as disappearance of the band corresponding to monomeric, SDS-soluble HttEx1Q45 and Q45 and concomitant appearance of SDSinsoluble material accumulated at the top of the gel using $13 \%$ Tris-tricine (Q45) or $12 \%$ Tris-glycine (HttEx1Q45) gels stained with SYPRO Orange (Invitrogen, Paisley, UK) followed by imaging with a LAS-3000 (Fujifilm, Tokyo, Japan) using the software Multigauge (Life Science Systems), for quantification of protein bands. Experiments were performed two to six times, and the data presented are representative of the experiments we performed.

Secondary structure prediction of DNAJB6 and sequence alignment

The sequences of human DNAJB6 (isoform B, sp|O751902) and DNAJB8 ( $\mathrm{sp} \mid \mathrm{Q} 8 \mathrm{NHS} 0)$, three ortogues to each of them, and human DNAJB1 (sp|P25685) were subjected to secondary structure prediction using several different programs (JPRED, APSSP, NetSurfP, PROF, SCRATCH, JUFO, PSIPRED, YASPIN, PORTER, and PROTPRED) at ExPASy website (http://www.expasy.org) and compared to the secondary structure determined for human DNAJB1, the N-terminal domain (PDB 1HDJ; Qian et al. 1996), and the C-terminal domain (PDB 3AGX; Suzuki et al. 2010). Sequence alignment was performed with Clustal Omega (www.ebi.ac.uk/Tools/msa/clustalo).

\section{Results}

DNAJB6 is an oligomeric protein

The DNAJB6 was recombinantly expressed in E. coli and purified by affinity chromatography (Fig. S1, Table S1). The His-tag was removed by cleavage with TEV protease, and when occasionally, the cleavage was incomplete; not only 
His-tagged DNAJB6 but also untagged DNAJB6 was retained on the column. This suggested that the purified DNAJB6 protein is not in a monomeric form, in consistence with the findings that in cells, DNAJB6 (Sarparanta et al. 2011) as DNAJB8 (Hageman et al. 2010) forms large oligomers. The oligomeric state of DNAJB6 was further analyzed by DLS (Fig. 1a), SEC (Fig. 1b), native PAGE (Fig. 1c), and negative stain EM (Fig. 1d), altogether revealing a polydisperse population of particles, with an average radius size of $12.7 \mathrm{~nm}$ (Table 1). The size distribution of the oligomers was similar for DLS measurements at temperatures from 10 to $40{ }^{\circ} \mathrm{C}$ and at concentrations ranging from 0.03 to $3 \mathrm{mg} / \mathrm{ml}$ (data not shown). Attempts to fractionate the heterogeneous DNAJB6 oligomers by SEC resulted in subpopulations of different size, as seen with native PAGE (Fig. 1c) and DLS (Table 1), which, however, still were heterogeneous. So, unlike what is known for most other DNAJB proteins that, generally, are thought to form dimers (Kampinga and Craig 2010; Cheetham and Caplan 1998; Li et al. 2009), DNAJB6 forms large and polydisperse oligomers.

\section{Assessment of PolyQ fibrillation}

The high propensity of polyQ to form $\beta$-sheet-rich fibrils is documented by different experimental techniques, including
Table 1 Size determination of DNAJB6 oligomers by DLS

\begin{tabular}{lllll}
\hline SEC fraction & $\begin{array}{l}\text { Radius size } \\
(\mathrm{nm})\end{array}$ & SD & $\begin{array}{l}\text { MW } \\
(\mathrm{kDa})\end{array}$ & SD \\
\hline Unfractionated & 12.7 & 4.7 & 1,280 & 478 \\
3 & 13.2 & 3.3 & 1,400 & 352 \\
6 & 10.8 & 2.8 & 880 & 231 \\
9 & 10.4 & 2.5 & 810 & 196 \\
\hline
\end{tabular}

Values are presented from DLS measurements of DNAJB6 oligomers, without (unfractionated) and with (fractionated: 3, 6, and 9) prior fractionation by SEC (Fig. 1b). Same fractions were also analyzed by native PAGE (Fig. 1c)

CD, EM, and X-ray diffraction (Papaleo and Invernizzi 2011). That fibrils bind the fluorescent dye ThT (Krebs et al. 2005) is underlying one of the most widely used tools in the study of amyloid fibrils (Biancalana and Koide 2010; Wu et al. 2010). We evaluated the effect of DNAJB6 on fibrillation, with polyQ as a peptide (Qn) and in the context of HttEx1 (HttEx1Qn), with $Q=15$ or 45 . The sequences were GAMKSF(Q)nF and GAMATLEKLMKAFESL KSF(Q)nPPPPPPPPPPPQLPQPPPQAQPLLPQPQPPPPP PPPPPGPAVAEEPLHRPGSLHHHHHH (with residues in HttEx1 underlined), resulting in molecular mass 6.5 and
Fig. 1 DNAJB6 forms large heterogeneous oligomers. Purified DNAJB6 protein was analyzed by DLS (a) and SEC (b), with molecular weight marker in gray dashed line. Fractions of the oligomeric DNAJB6 peak, of which three are marked 3,6 , and 9 , were further analyzed with native PAGE (c) where $M W$ molecular standard, lane 1 unfractionated sample, and lanes 2-10 SEC fractions. The fractions marked with an asterisk were also analyzed with DLS (Table 1), and fraction 6 was stained with uranyl acetate for negative stain EM (d) with a scale bar corresponding to $100 \mathrm{~nm}$ a

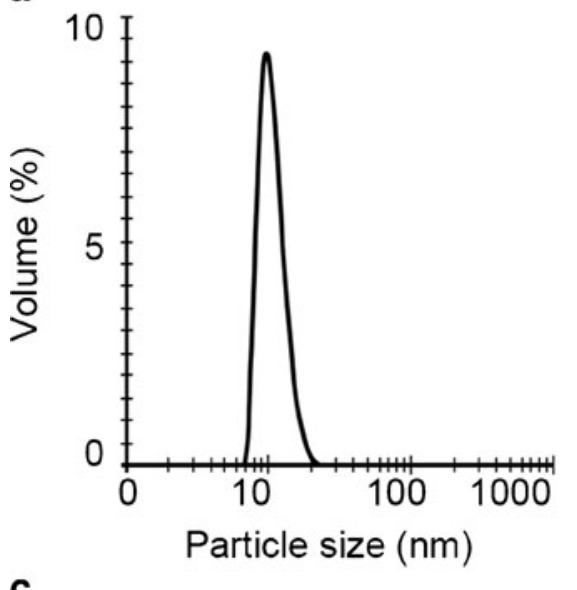

C

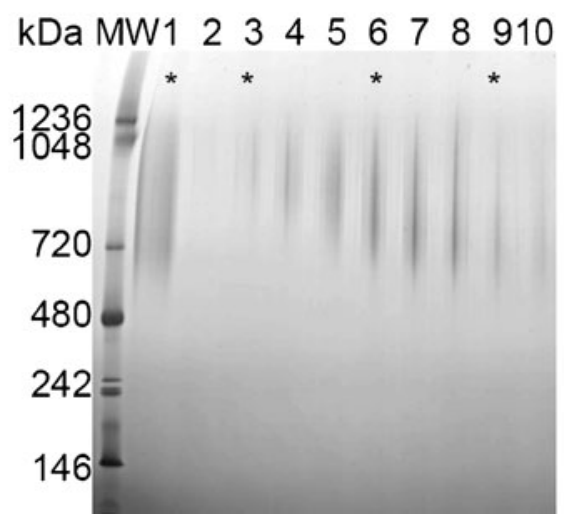

b

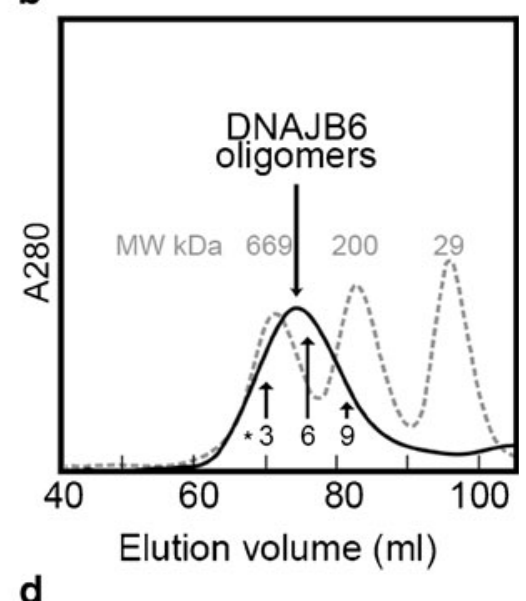

d

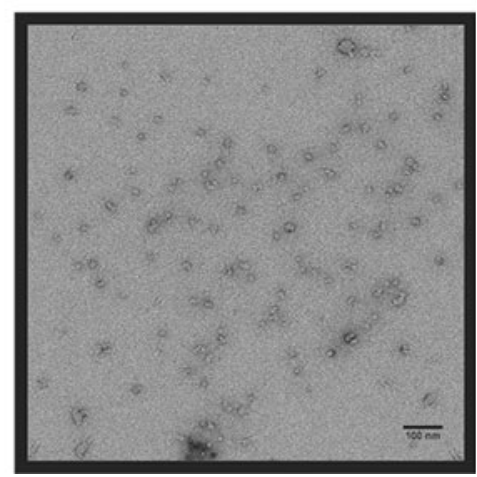


Fig. 2 Concentration-dependent polyQ fibrillation. Fibrillation was measured as the ThT fluorescence increase, and triplicate samples are shown for Q45 peptide (a) and for HttEx1Q45 (b), at protein concentrations $6.4,8,10,12.8$, 16 , and $20 \mu \mathrm{M}$, color coded with increasingly darker blue indicating increasing concentration. The difference in absolute fluorescence intensity between Q45 and HttEx1Q45 is arbitrary and gives no quantitative comparison of their extent of fibrillation. Half times of fibrillation $\left(t_{0.5}\right.$ values, Table 2) were extracted from the curves in $\mathbf{a}$ and $\mathbf{b}$ and $\log t_{0.5}$ plotted against the log concentration of Q45 peptide and HttEx1Q45, respectively (c). Samples of polyQ peptide (d, e) and HttEx1 (f, g) with Q15 (d, f) or Q45 $(\mathbf{e}, \mathbf{g})$ were visualized with TEM to confirm fibril formation. Scale bars correspond to $200 \mathrm{~nm}$
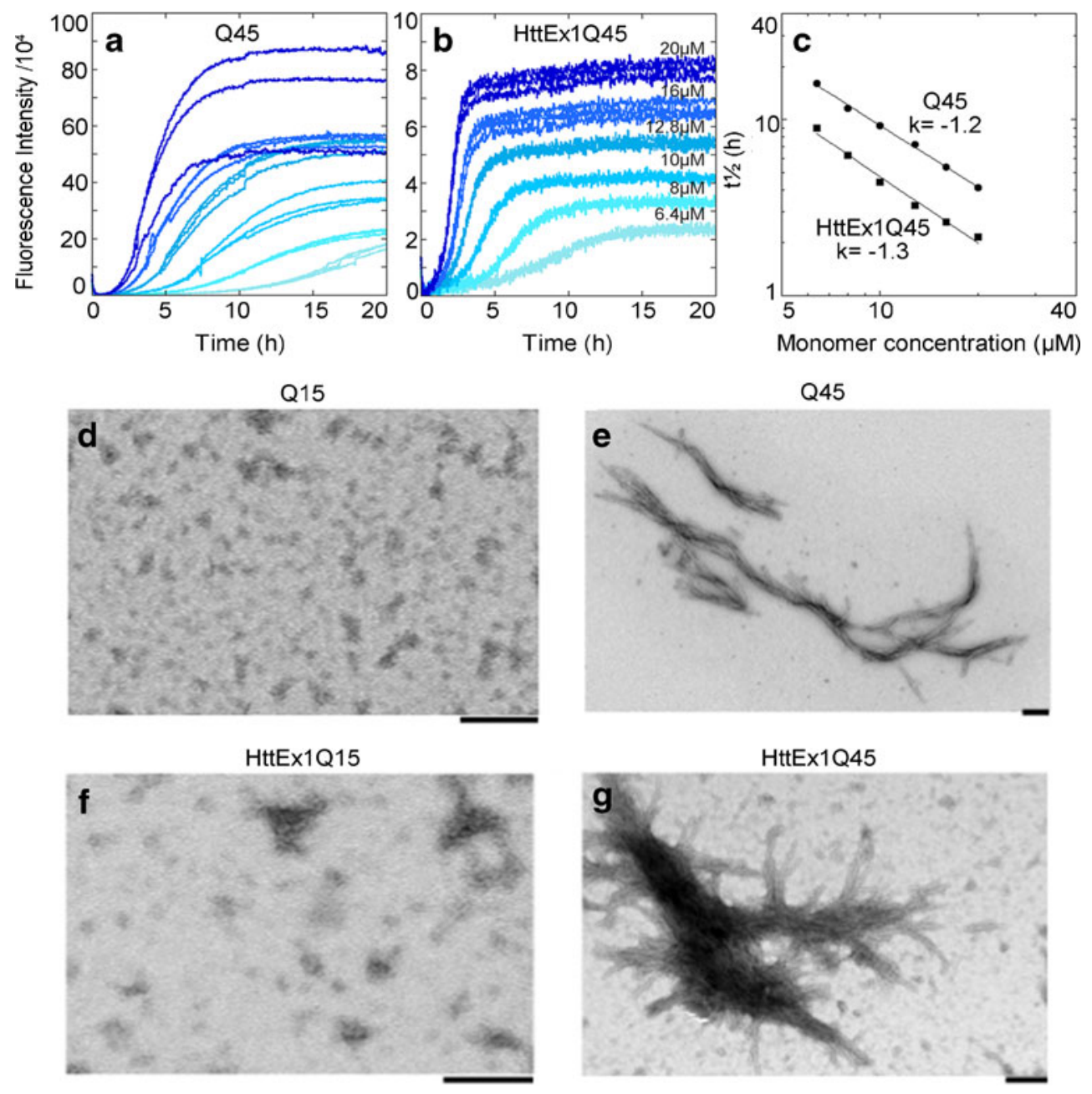

$14 \mathrm{kDa}$, respectively, for $n=45$. Since the constructs are highly prone to aggregation, they were expressed and purified as fusions with MBP (Fig. S2). Fibrillation was initiated by addition of TEV protease, which starts the release of the fibrillation-prone polyQ monomers from MBP, and was monitored by continuous measurement of ThT fluorescence. For both Q45 (Fig. 2a) and HttEx1Q45 (Fig. 2b), fibrillation occurred in a concentration-dependent manner, as previously reported (Wetzel 2012). To yield information on the dominating fibrillation pathway (Cohen et al. 2012), half times of fibrillation were extracted (Table 2) from the curves in Fig. $2 \mathrm{a}$, b. A power function was fitted to $t_{0.5}$ versus concentration $c\left(t_{0.5}=C \times c^{k}\right)$, where $C$ is a constant, and $k$ is the slope in the log-log plot (Fig. 2c). Assuming a dimer as critical nucleus, these $k$ values for Q45 and HttEx1Q45 (-1.2 and -1.3 , respectively) suggest that the fibrillation was largely dependent on the so-called primary nucleation (Cohen et al. 2012). No increase in ThT fluorescence was observed neither for Q15 nor HttEx1Q15 (data not shown). In agreement with this, no fibrils were observed by for Q15 nor HttEx1Q15 (Fig. 2d, f), whereas the increase in ThT fluorescence intensity recorded both for Q45 and HttEx1Q45 correlated with the appearance of fibrils (Fig. 2e, g).

Suppression of polyQ fibrillation by DNAJB6 Having established a robust fibrillation assay for Q45 and HttEx1Q45,

Table 2 Concentration-dependent PolyQ fibrillation

\begin{tabular}{llllll}
\hline Concentration & \multicolumn{2}{l}{$\mathrm{Q} 45$} & & \multicolumn{2}{l}{ HttEx1Q45 } \\
\cline { 2 - 3 } \cline { 5 - 6 }$(\mu \mathrm{M})$ & $t_{0.5}(\mathrm{~h})$ & $\mathrm{SD}$ & & $t_{0.5}(\mathrm{~h})$ & $\mathrm{SD}$ \\
\hline 6.4 & 16.0 & 0.2 & & 8.9 & 0.3 \\
8 & 11.6 & 0.2 & 6.3 & 0.0 \\
10 & 9.2 & 0.1 & 4.4 & 0.1 \\
12.8 & 7.2 & 0.3 & 3.3 & 0.1 \\
16 & 5.3 & 0.0 & 2.6 & 0.1 \\
20 & 4.1 & 0.1 & 2.2 & 0.0 \\
\hline
\end{tabular}

Fibrillation was measured as ThT fluorescence increase at various concentrations of polyQ. The half time $\left(t_{0.5}\right)$ for fibrillation was extracted from the curves in Fig. 2a (Q45) and Fig. 2b (HttEx1Q45), respectively, and plotted in Fig. 2c 

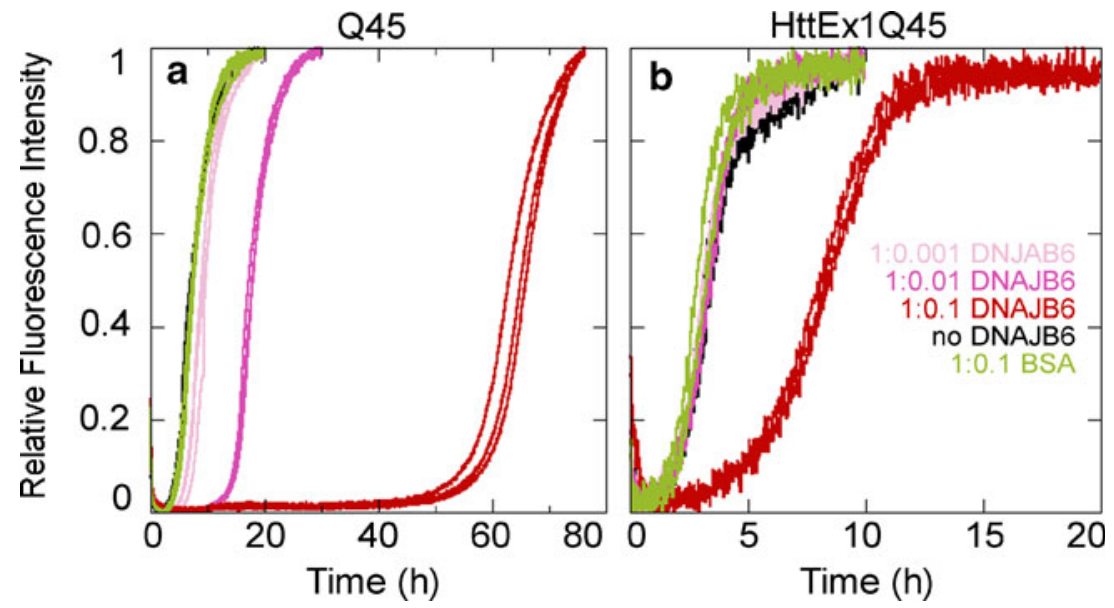

Fig. 3 Suppression of fibrillation of Q45 and HttEx1Q45 by DNAJB6. Fibrillation was measured in triplicates as the relative ThT fluorescence increase, at $15 \mu \mathrm{M}$ Q45 (a) or HttEx1Q45 (b) with DNAJB6 added in varying polyQ to DNAJB6 molar ratios: 1:0.1 (red), 1:0.01 (dark pink),

1:0.001 (pink), no added DNAJB6 (black), and BSA added in ratio 1:0.1 (yellow green). Note the difference in time scale between Q45 and HttEx1Q45. Molar ratios were calculated as monomer to monomer, although DNAJB6 is oligomeric

we next assessed the effect of DNAJB6 on fibrillation. The lag phase preceding fibrillation increased with increasing concentrations of DNAJB6 (Fig. 3). For Q45 (Fig. 3a), fibrillation suppression occurred at a molar ratio polyQ to DNAJB6 as low as 1:0.01. For HttEx1Q45, the fibrillation suppression was less efficient (Fig. 3b). Suppression can be estimated as the increase in the $t_{0.5}$ values for fibrillation and was increased by DNAJB6 approximately tenfold for Q45 at a 1:0.1 M ratio, but only by a factor of 3 for HttExQ45 at a similar molar ratio. At lower molar ratio (1:0.01), the $t_{0.5}$ of fibrillation was increased by a factor of 2 for Q45, while no significant effects were seen for HttEx1Q45.
Suppression of polyQ fibrillation by DNAJB6 in presence of preformed fibrils To investigate the mechanism of fibrillation suppression further, we performed experiments under seeded conditions to mimic later stages where some nuclei have been formed already, and elongation of fibrils and secondary nucleation processes may occur (Cohen et al. 2012). Fibrillation suppression by added DNAJB6 was evaluated without seeding (primary nucleation dominates) and by seeding with preformed fibrils at two concentrations ( $5 \%$, secondary nucleation may dominate, and $20 \%$, the elongation of fibrils dominates), for Q45 (Fig. 4a) and HttEx1Q45 (Fig. 4b). The suppression was largest under non-seeded
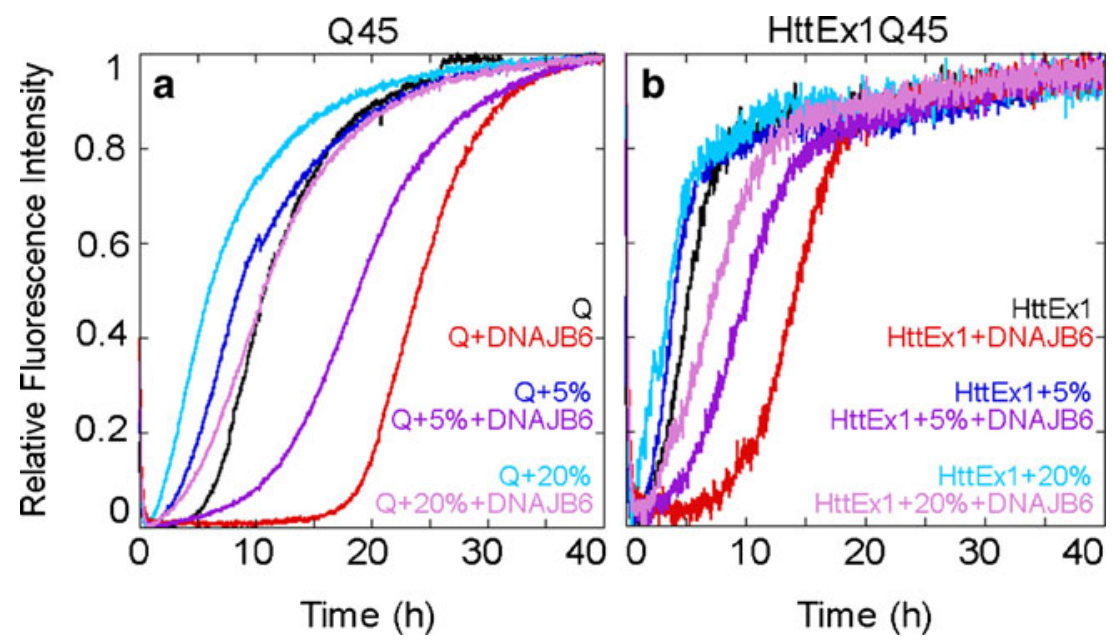

Fig. 4 Suppression of fibrillation of Q45 and HttEx1Q45 by DNAJB6 in the presence of preformed fibrils. Fibrillation was measured as the relative ThT fluorescence increase at $10 \mu \mathrm{M}$ Q45 (a) or HttEx1Q45 (b) under different seeding conditions: no seed (black) without and with DNAJB6 (red), seeding with $5 \%$ preformed fibrils (dark blue) without and with

DNAJB6 (purple), seeding with $20 \%$ preformed fibrils (light blue) without and with DNAJB6 (pink), with preformed fibrils taken after $20 \mathrm{~h}$ of incubation according to Fig. 3. The molar ratio between chaperone and polyQ was 1:0.01 for Q45 and 1:0.1 for HttEx1. Molar ratios were calculated as monomer to monomer, although DNAJB6 is oligomeric 
conditions, but not insignificant under seeded conditions. Thus, there is clearly an effect of DNAJB6 not only on the primary nucleation but also on later stages of fibrillation.

Suppression of the formation of SDS-insoluble aggregates by DNAJB6 As a complement to the evaluation of polyQ aggregation as the formation of fibrils by ThT fluorescence measurements, we also followed the polyQ aggregation by continuous withdrawal of samples for SDS-PAGE and assessment of the distribution of protein between bands representing the monomeric forms of Q45 and HttEx1Q45, respectively (Fig. 5a, c) and bands representing SDS-insoluble aggregates at the top of the gel (Fig. 5b, d). Quantification of the band intensities after imaging showed disappearance of monomeric forms (Fig. 5e, f) and concomitant appearance of SDSinsoluble aggregates (Fig. 5g, h), in approximately $24 \mathrm{~h}$ for Q45 and $1 \mathrm{~h}$ for HttEx1Q45. Thus, for Q45, the formation of ThT-binding fibrils (Fig. 3a) preceded the formation of SDSinsoluble aggregates (Fig. 5c), whereas for HttEx1Q45, the formation of ThT-binding fibrils (Fig. 3b) and aggregates
Fig. 5 Suppression of the formation of SDS-insoluble aggregates by DNAJB6.

Formation of SDS-insoluble polyQ aggregates was followed on SDS-PAGE for $20 \mu \mathrm{M}$ Q45 $(\mathbf{a}, \mathbf{c})$ and Ex1Q45 $(\mathbf{b}, \mathbf{d})$ in the absence $(\mathbf{a}, \mathbf{b})$ or presence $(\mathbf{c}, \mathbf{d})$ of $20 \mu \mathrm{M}$ DNAJB6. Gels were stained for $1 \mathrm{~h}(\mathbf{b}, \mathbf{d})$ or $3 \mathrm{~h}(\mathbf{a}, \mathbf{c})$ with SYPRO Orange. This dye is poorly bound to $\mathrm{HttEx} 1 \mathrm{Q} 45$ and even more so to Q45, which appear as faintly stained bands on the gels, despite being present at much higher levels than DNAJB6. Protein band intensities were quantified and plotted as a relative amount of soluble monomers $(\mathbf{e}, \mathbf{f})$ or SDSinsoluble aggregates $(\mathbf{g}, \mathbf{h})$ at $20 \mu \mathrm{M}$ Q45 (e, g) and HttEx1Q45 (f, h) without DNAJB6 (filled circles) or with DNAJB6 in a molar ratio of 1:0.1 (open diamonds) or 1:1 (filled squares). Molar ratios were calculated as monomer to monomer, although DNAJB6 is oligomeric. Note the difference in time scale between Q45 and HttEx1Q45. The data were fitted to the sigmoidal equation $y=a$ / $(1+\exp (b(c-x)))$, where $a$ is the proportion of aggregates at steady state, $b$ is the slope of the exponential growth phase, and $c$ is the time where $50 \%$ of the aggregation is reached a
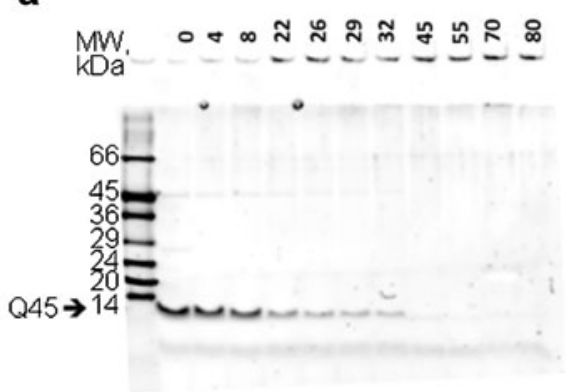

C

Time (h)
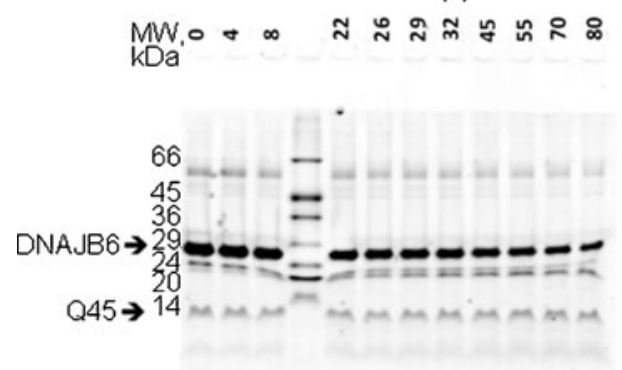

e

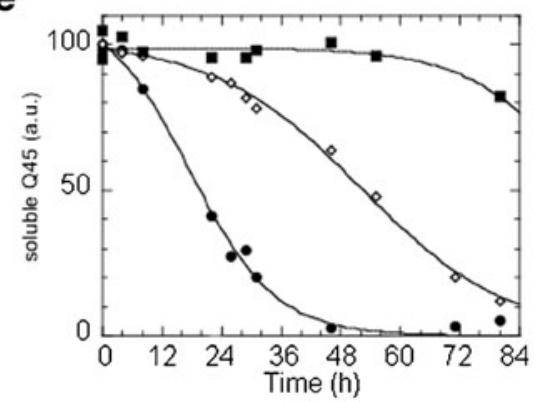

g

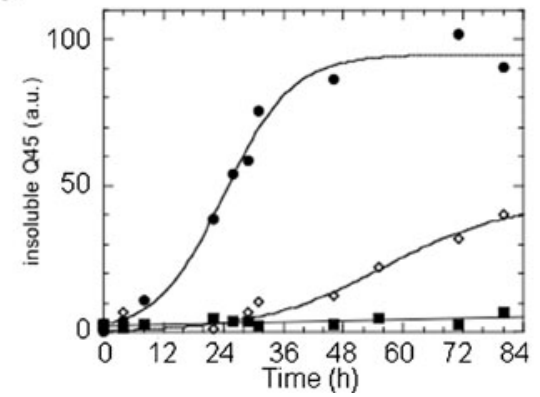

b
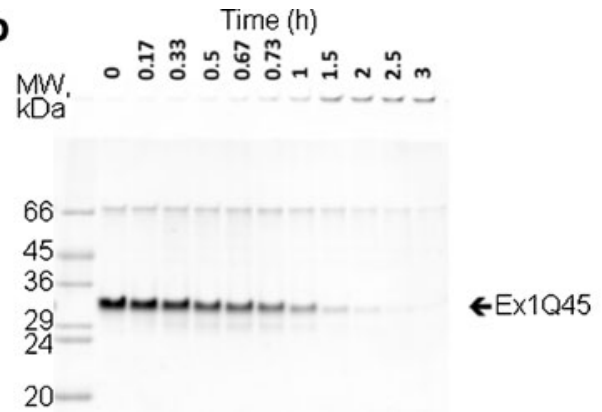

d Time (h)
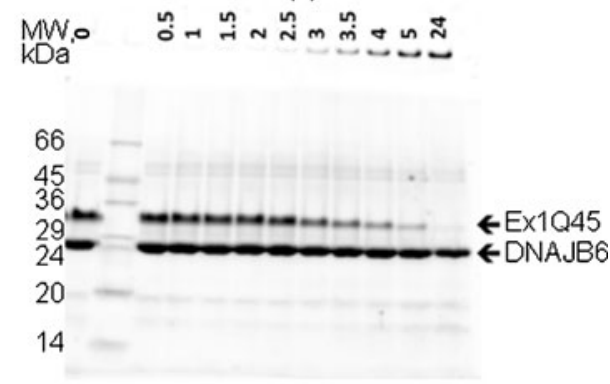

f

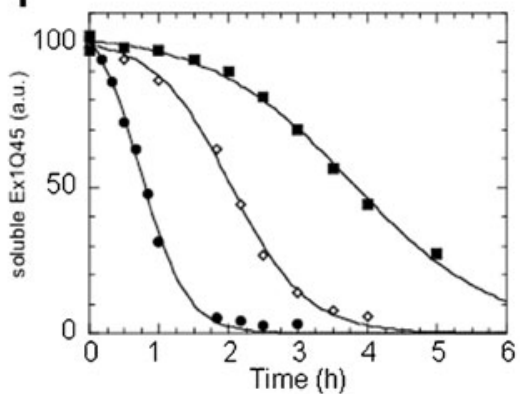

h

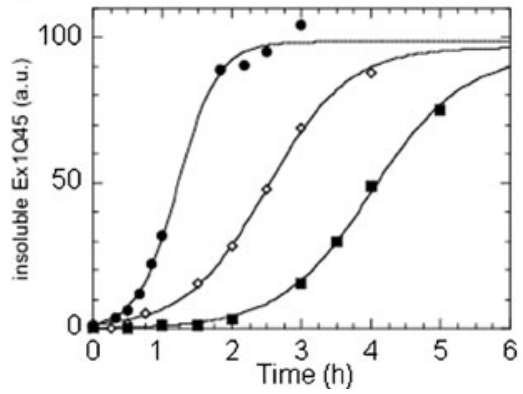



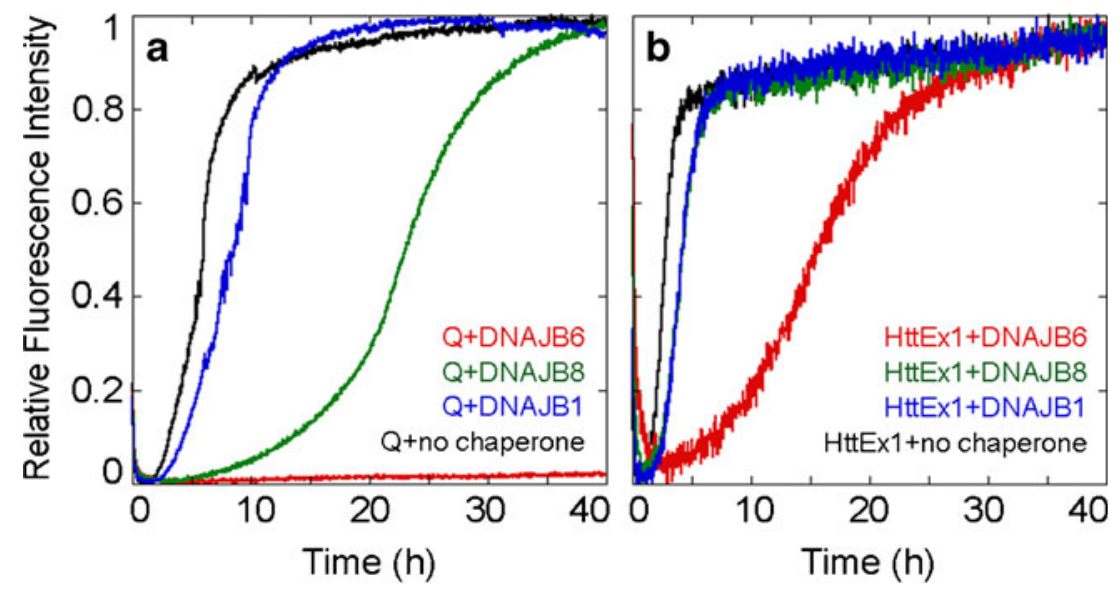

Fig. 6 Specificity in suppression of fibrillation by DNAJB6. Fibrillation was measured as the relative ThT fluorescence increase, and incubation was made at $15 \mu \mathrm{M}$ Q45 (a) and HttEx1Q45 (b) with no chaperone (black), DNAJB6 (red), DNAJB8 (green), and DNAJB1

(blue). The monomeric molar ratio between chaperone and polyQ was 1:0.1. Molar ratios were calculated as monomer to monomer, although DNAJB6 is oligomeric

(Fig. 5d) occurred rather simultaneously and more rapidly than for Q45. This is consistent with previous observations of peptides relative to the exon 1 of huntingtin, where the Nterminal 17 amino acids cause an initial aggregation prior to rearrangement and fibril formation (Papaleo and Invernizzi 2011). Equimolar amounts of DNAJB6 fully prevented aggregation of Q45 during $84 \mathrm{~h}$ (Fig. 5a, c), and delayed it fourfold for HttEx1Q45 (Fig. 5b, d). With substoichiometric amounts of DNAJB6 (1:0.1), the monomeric form of Q45 and HttEx1Q45 disappeared, and the same amount of SDS-insoluble aggregates appeared in case of HttEx1Q45, but not for Q45 (Fig. 5d, compared to c). Hence, even substoichiometric amounts of DNAJB6 interact with Q45 monomers and counteract the appearance of insoluble aggregates.
Fig. 7 Suppression of fibrillation by DNAJB6 with and without HSPA1. Fibrillation was measured as the relative ThT fluorescence increase, and incubation was made of $15 \mu \mathrm{M}$ Q45 (a, b) and HttEx1Q45 (c, d) with no chaperone in the absence (black) or presence (gray) of HSPA1, with DNAJB6 in the absence (red) or presence (pink) of HSPA1, and with DNAJB1 in the absence (dark blue) or presence (light blue) of HSPA1. The monomeric molar ratio between chaperone and polyQ was 1:0.01. Molar ratios were calculated as monomer to monomer, although DNAJB6 is oligomeric
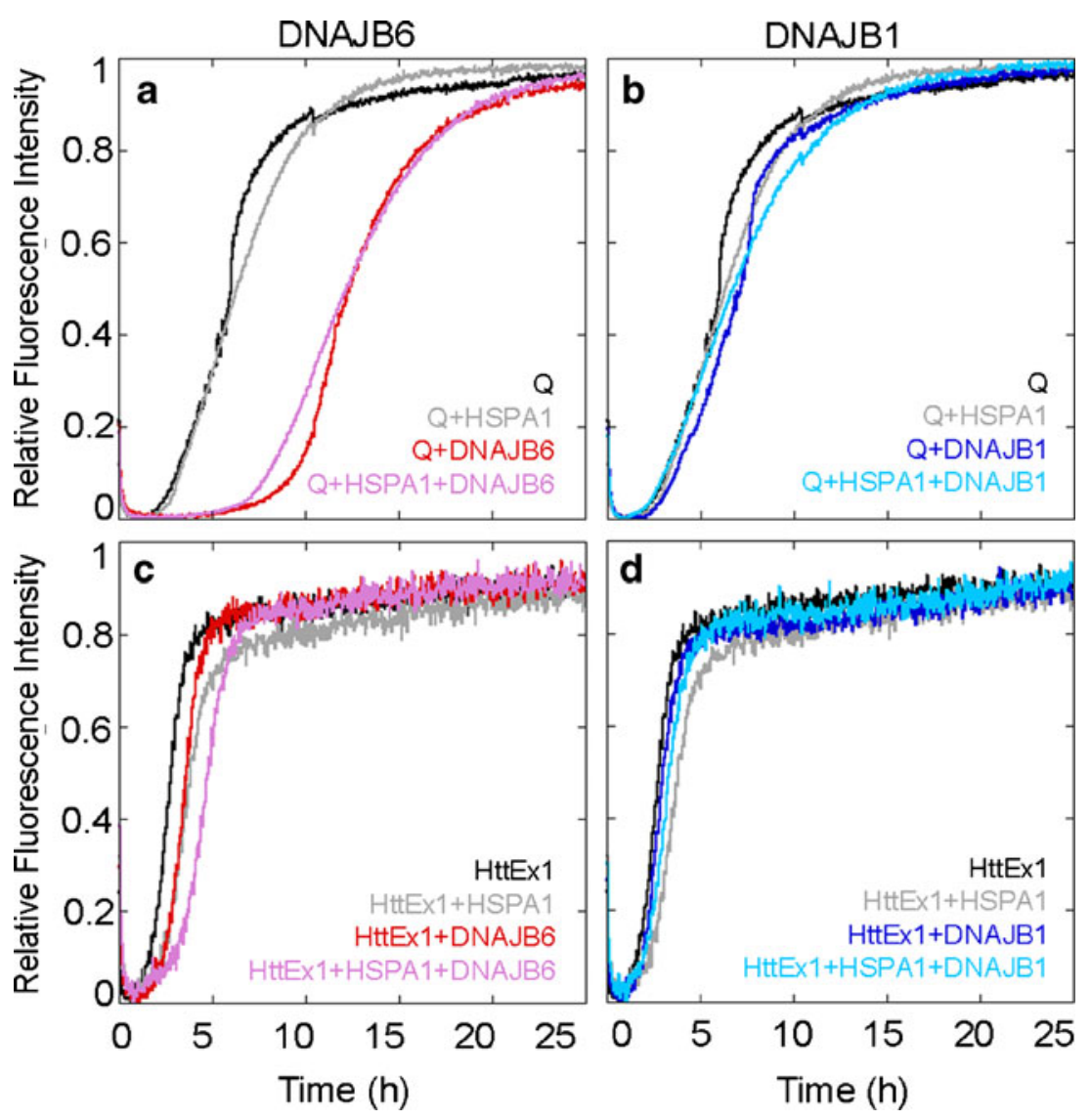
Fibrillation suppression by DNAJB6 compared to other $D N A J B$ proteins To evaluate how specific the polyQ fibrillation suppression by DNAJB6 is compared to other DNAJB proteins, we added DNAJB6, its close homologue DNAJB8, and the more canonical family member, the structurally characterized homologue DNAJB1. DNAJB6 suppressed fibrillation of Q45 entirely, DNAJB8 delayed fibrillation by a factor of 4, and DNAJB1 delayed fibrillation only slightly, at a molar ratio 1:0.1 (Fig. 6a). At a molar ratio 1:0.01 where DNAJB1 had no effect at all, DNAJB6 still delayed fibrillation with nearly a factor 3 (data not shown). For HttExQ45, the fibrillation was delayed 2.5-fold with DNAJB6 and, to a small extent, by DNAJB1 and DNAJB8 (Fig. 6b). The suppression of polyQ fibrillation by DNAJB6 was not further enhanced in presence of HSPA1 and ATP (Fig. 7), confirming the finding in cellular systems that suppression of polyQ aggregation by DNAJB6 is HSPA1/ATP independent (Hageman et al. 2010; Gillis et al. 2013). Thus, DNAJB6 has unique fibrillation suppression properties, which are lacking in DNAJB1 and, probably, are located in the C-terminal and putatively substrate-binding domain which is not very conserved among the many different DNAJB proteins. The sequence similarity between DNAJB6 and DNAJB1 is largest in the N-terminal J domain (59\% sequence identity) and in the C-terminal domain that has been structurally characterized for DNAJB1, showing two $\beta$-sandwich folds each with $2+3$ antiparallel $\beta$-strands and one $\alpha$-helix and $\alpha$-helical dimerization domain (Suzuki et al. 2010; Hu et al. 2008), and the sequence identity is too low $(17 \%)$ for homology modeling. However, secondary structure predictions and sequence alignment (Fig. S3), with the structure determined for DNAJB1 correlating well with the prediction for DNAJB1, suggest that DNAJB6 and DNAJB8 have one $\beta$-sandwich fold in the $\mathrm{C}$-terminal domain (Fig. 8).

\section{Discussion}

Here, we have shown that the purified DNAJB6 protein forms heterogeneous oligomers that cannot be separated into more homogeneous fractions (Fig. 1, Table 1). This resembles the polydisperse and dynamic oligomerization of some small heat shock proteins for example $\alpha B$-crystallin (Baldwin et al. 2011). DNAJB6 efficiently suppressed fibrillation of Q45 and, less efficiently, suppressed fibrillation of HttEx1Q45 (Figs. 3, 4, and 5). Furthermore, DNAJB6 was undoubtedly more efficient in suppressing fibrillation than DNAJB1 and independent on the presence of HSPA1 and ATP (Figs. 6 and 8). These data obtained with purified proteins provide evidence in support of the effects previously observed in cells, when overexpression of DNAJB6 was combined with expression of GFP-labeled huntingtin exon 1 (Hageman et al. 2010) and polyQ peptides (Gillis et al. 2013).

DNAJB6 was identified, together with its closest homologue DNAJB8, as unique polyQ aggregation suppressors among the human HSPA, HSPH, and DNAJ family members (Hageman et al. 2010). The suppression was largely independent of the expression of Hsp70 members. The absence of a comparable polyQ aggregation inhibitory effect of DNAJB1 that shares a homologous $\mathrm{J}$ domain but has little sequence resemblance with DNAJB6 in its C-terminus (only $17 \%$ ) supports the crucial role for the C-terminal domain of DNAJB6 and DNAJB8 in the polyQ aggregation suppression. The DNAJB1 C-terminus lacks a serine-rich motif(SSF-SST) and two lysines that were found to be important for the antiaggregation function (Hageman et al. 2010) (Fig. 8).

DNAJB6 was more efficient in suppressing fibrillation of polyQ peptides than $\mathrm{HttEx} 1$ with polyQ stretches of identical

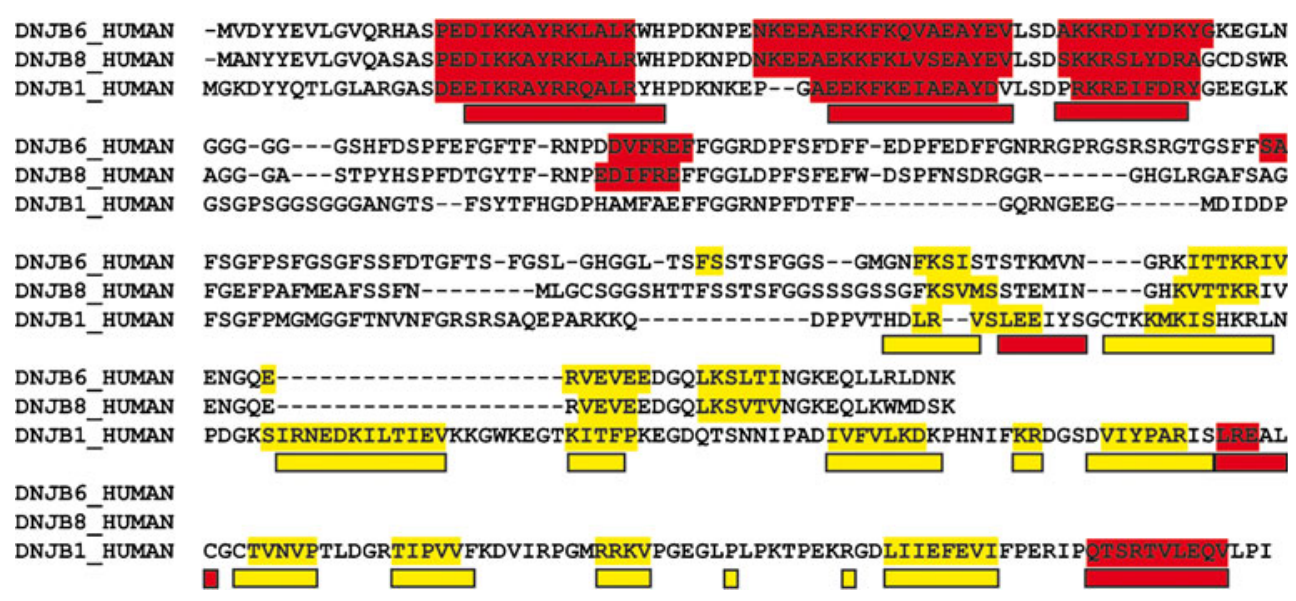

Fig. 8 Secondary structure prediction to assess the C-terminal domain of DNAJB6. Secondary structure prediction, with predicted $\alpha$-helices in red, predicted $\beta$-strands in yellow for human DNAJB6b (isoform B, $\mathrm{sp} \mid \mathrm{O} 75190-2)$, its closest paralogue DNAJB8 (sp|Q8NHS0), and its more distantly related paralogue, DNAJB1 (sp|P25685) for which the

structure has been determined to atomic resolution [PDB:1HDJ for the N-terminal domain (Qian et al. 1996), PDB:3AGX for the C-terminal domain (Suzuki et al. 2010)], with $\alpha$-helices as red and $\beta$-strands as yellow boxes below the aligned sequences 
length, as assessed by ThT fluorescence (Fig. 3) and the formation of SDS-insoluble aggregates (Fig. 5). There may be several reasons for this. The fibrillation-promoting properties of the polyQ-flanking regions within HttEx 1 might counteract DNAJB6-mediated inhibition, or these regions might disfavor the interaction between DNAJB6 and polyQ. The polyQ context, specifically the $17 \mathrm{~N}$-terminal amino acids in HttEx1, significantly increases polyQ fibrillation propensity (Wetzel 2012); (Papaleo and Invernizzi 2011), which could make it more difficult to counteract fibrillation. The inhibition of polyQ aggregation by DNAJB6 anyway appears to be largely context independent with the polyQ stretch as the only common denominator, since DNAJB6 was also very efficient at suppressing aggregation of other polyQ-containing proteins, like ataxin-3 and the androgen receptor (Hageman et al. 2010).

So, how can our current findings of highly efficient suppression of polyQ peptide aggregation by DNAJB6 be related to its anti-aggregation and toxicity-preventing effects in cellular (Hageman et al. 2010; Chuang et al. 2002) and animal models (Hageman et al. 2010; Fayazi et al. 2006) of polyQ diseases? One obvious possibility relates to the findings that proteins containing expanded polyQ stretches are not fully degraded by the proteasome (Venkatraman et al. 2004). As a consequence, polyQ peptides that have a high propensity to aggregate into fibrillar assemblies (Wetzel 2012) are released from the proteasome during the degradation of huntingtin (Holmberg et al. 2004; Venkatraman et al. 2004) and other polyQ proteins. That free polyQ peptides are not readily detected in cells can be due to their immediate capture by DNAJB6-like chaperones and subsequent degradation by peptidases or autophagy (Menzies et al. 2010). If such processing fails, polyQ peptides would, however, become enriched and immediately seed aggregation into both main and side chain hydrogen-bonded fibrillar structures (Berryman et al. 2009; Streets et al. 2013). Other proteins containing $\mathrm{Q}$ stretches or protease-generated fragments of the polyQ proteins next will co-aggregate with such peptides and contribute to the toxicity by either direct aggregated toxicity or (secondary) loss of function of these sequestered proteins. The rate and propensity of this will likely depend on the context around the polyQ stretches in these fragments (Dehay and Bertolotti 2006). If released in the cellular environment upon cell death, aggregates can be transmitted to neighboring cells and act as seeds promoting the aggregation of endogenous polyQ-containing proteins (Ren et al. 2001), reminiscent of the spreading seen for the prion PrP (Brundin et al. 2010). To mimic proteasomal release of polyQ peptides in living cells, overexpression of genetically engineered constructs of polyQ peptides was therefore performed, showing that the polyQ peptides induced the formation of aggregates in which polyQ-containing proteins and other heat shock proteins (HSPs) were detected, supporting the view that polyQ peptides can act as seeds for aggregation (Raspe et al. 2009).
Moreover, using the same cell system, it was recently shown that co-expression of these genetically engineered constructs of polyQ peptides with DNAJB6 suppressed aggregation (Gillis et al. 2013). Interestingly, in the few aggregates that did form, DNAJB6 and DNAJB8 were found in the core of the aggregates, whereas other chaperones (HSPA1) only associated with the periphery of the aggregates. This is indicating that DNAJB6 interacts early and directly with polyQ peptides as shown in this study, and the other HSPs interact later and more indirectly, presumably not until after the aggregates have formed.

To summarize, the data presented in this study show that DNAJB6 can directly bind to and highly efficiently suppress polyQ peptide fibrillation initiated by soluble, pure polyQ peptides. This is in line with recent cellular findings that DNAJB6 is a very strong protector against protein toxicity associated with polyQ aggregation diseases. The view of DNAJB6 as a peptide-binding chaperone that can sweep up polyQ peptides that are incompletely degraded by and released from the proteasome and suppress fibrillation initiation makes DNAJB6 an interesting target for therapy against such protein aggregation diseases.

Acknowledgments Eva Thulin is warmly acknowledged for recombinant protein expression and Professor Sara Linse for valuable advice on the fibrillation assays. Dr. Jurre Hageman is thanked for his help with the initial cloning work and Luc Bousset for the polyQ and HttEx1 expression constructs and helpful discussions. Financial support is provided from the Magnus Bergvall Foundation and the Carl Tryggers Foundation (CTS 12:117). The Research School of Pharmaceutical Sciences and Kungliga Fysiografiska Sällskapet at Lund University are thanked for their financial support to CM. The Kampinga lab is supported for the DNAJB work through grants form Agentschap.nl (IOP IGE07004). The Melki lab is supported through grants from the Human Frontier Science Program, the Agence Nationale de la Recherche (ANR-09-MNPS-01301), the Centre National de la Recherche Scientifique, and the Fondation Bettencourt Schueller.

Open Access This article is distributed under the terms of the Creative Commons Attribution License which permits any use, distribution, and reproduction in any medium, provided the original author(s) and the source are credited.

\section{References}

Baldwin AJ, Lioe H, Robinson CV, Kay LE, Benesch JLP (2011) $\alpha$ BCrystallin polydispersity is a consequence of unbiased quaternary dynamics. J Mol Biol 413(2):297-309. doi:10.1016/j.jmb.2011. 07.016

Berke SJ, Schmied FA, Brunt ER, Ellerby LM, Paulson HL (2004) Caspase-mediated proteolysis of the polyglutamine disease protein ataxin-3. J Neurochem 89(4):908-918. doi:10.1111/j.1471-4159. 2004.02369.x

Berryman JT, Radford SE, Harris SA (2009) Thermodynamic description of polymorphism in Q- and N-rich peptide aggregates revealed by atomistic simulation. Biophys J 97(1):1-11. doi:10. 1016/j.bpj.2009.03.062 
Biancalana M, Koide S (2010) Molecular mechanism of thioflavin-T binding to amyloid fibrils. Biochim Biophys Acta 1804(7):14051412. doi:10.1016/j.bbapap.2010.04.001

Bieschke J, Herbst M, Wiglenda T, Friedrich RP, Boeddrich A, Schiele F, Kleckers D, del Amo JML, Gruening BA, Wang Q, Schmidt MR, Lurz R, Anwyl R, Schnoegl S, Faendrich M, Frank RF, Reif B, Guenther S, Walsh DM, Wanker EE (2012) Small-molecule conversion of toxic oligomers to nontoxic beta-sheet-rich amyloid fibrils. Nat Chem Biol 8(1):93-101. doi:10.1038/nchembio.719

Bradford MM (1976) A rapid and sensitive method for the quantitation of microgram quantities of protein utilizing the principle of protein-dye binding. Anal Biochem 72(1/2):248-254

Brundin P, Melki R, Kopito R (2010) Prion-like transmission of protein aggregates in neurodegenerative diseases. Nat Rev Mol Cell Biol 11(4):301-307. doi:10.1038/nrm2873

Cheetham ME, Caplan AJ (1998) Structure, function and evolution of DnaJ: conservation and adaptation of chaperone function. Cell Stress Chaperones 3(1):28-36. doi:10.1379/1466-1268(1998) $003<0028$ :sfaeod $>2.3$. co; 2

Chuang J-Z, Zhou H, Zhu M, Li S-H, Li X-J, Sung C-H (2002) Characterization of a brain-enriched chaperone, MRJ, that inhibits huntingtin aggregation and toxicity independently. J Biol Chem 277(22):19831-19838. doi:10.1074/jbc.M109613200

Cohen SIA, Vendruscolo M, Dobson CM, Knowles TPJ (2012) From macroscopic measurements to microscopic mechanisms of protein aggregation. J Mol Biol 421(2-3):160-171. doi:10.1016/j.jmb. 2012.02.031

de Almeida LP, Ross CA, Zala D, Aebischer P, Deglon N (2002) Lentiviral-mediated delivery of mutant huntingtin in the striatum of rats induces a selective neuropathology modulated by polyglutamine repeat size, huntingtin expression levels, and protein length. J Neurosci 22(9):3473-3483

Dehay B, Bertolotti A (2006) Critical role of the proline-rich region in huntingtin for aggregation and cytotoxicity in yeast. J Biol Chem 281(47):35608-35615. doi:10.1074/jbc.M605558200

Fayazi Z, Ghosh S, Marion S, Bao X, Shero M, Kazemi-Esfarjani P (2006) A Drosophila ortholog of the human MRJ modulates polyglutamine toxicity and aggregation. Neurobiol Dis 24(2):226-244. doi:10.1016/j.nbd.2006.06.015

Gatchel JR, Zoghbi HY (2005) Diseases of unstable repeat expansion: mechanisms and common principles. Nat Rev Genet 6(10):743755. doi: $10.1038 / \operatorname{nrg} 1691$

Gestwicki JE, Garza D (2012) Protein quality control in neurodegenerative disease. Prog Mol Biol Transl Sci 107:327-353. doi:10. 1016/b978-0-12-385883-2.00003-5

Gillis J, Schipper-Krom S, Juenemann K, Gruber A, Coolen S, van Nieuwendijk R, van Veen H, Overkleeft H, Goedhart J, Kampinga HH, Reits EA (2013) The DNAJB6 and DNAJB8 chaperones prevent intracellular aggregation of polyglutamine peptides. J Biol Chem. doi:10.1074/jbc.M112.421685

Graham RK, Deng Y, Slow EJ, Haigh B, Bissada N, Lu G, Pearson J, Shehadeh J, Bertram L, Murphy Z, Warby SC, Doty CN, Roy S, Wellington CL, Leavitt BR, Raymond LA, Nicholson DW, Hayden MR (2006) Cleavage at the caspase-6 site is required for neuronal dysfunction and degeneration due to mutant huntingtin. Cell 125(6):1179-1191. doi:10.1016/j.cell.2006.04.026

Hageman J, Rujano MA, van Waarde MAWH, Kakkar V, Dirks RP, Govorukhina N, Oosterveld-Hut HMJ, Lubsen NH, Kampinga HH (2010) A DNAJB chaperone subfamily with HDACdependent activities suppresses toxic protein aggregation. Mol Cell 37(3):355-369. doi:10.1016/j.molcel.2010.01.001

Harris GM, Dodelzon K, Gong L, Gonzalez-Alegre P, Paulson HL (2010) Splice isoforms of the polyglutamine disease protein ataxin-3 exhibit similar enzymatic yet different aggregation properties. PLoS One 5(10):e13695. doi:10.1371/journal.pone.0013695
Holmberg CI, Staniszewski KE, Mensah KN, Matouschek A, Morimoto RI (2004) Inefficient degradation of truncated polyglutamine proteins by the proteasome. EMBO J 23(21):4307-4318. doi:10.1038/sj.emboj.7600426

Hu J, Wu Y, Li J, Qian X, Fu Z, Sha B (2008) The crystal structure of the putative peptide-binding fragment from the human $\mathrm{Hsp} 40$ protein Hdj1. BMC Struct Biol 8(1):3. doi:10.1186/1472-6807-8-3

Jung J, Xu K, Lessing D, Bonini NM (2009) Preventing ataxin-3 protein cleavage mitigates degeneration in a Drosophila model of SCA3. Hum Mol Genet 18(24):4843-4852. doi:10.1093/hmg/ddp456

Kampinga HH (1993) Thermotolerance in mammalian cells. Protein denaturation and aggregation, and stress proteins. J Cell Sci 104:11-17

Kampinga HH, Craig EA (2010) The HSP70 chaperone machinery: J proteins as drivers of functional specificity. Nat Rev Mol Cell Biol 11(8):579-592. doi:10.1038/nrm2941

Kar K, Jayaraman M, Sahoo B, Kodali R, Wetzel R (2011) Critical nucleus size for disease-related polyglutamine aggregation is repeat-length dependent. Nat Struct Mol Biol 18(3):328-336. doi:10.1038/nsmb.1992

Krebs MRH, Bromley EHC, Donald AM (2005) The binding of thioflavin-T to amyloid fibrils: localisation and implications. J Struct Biol 149(1):30-37. doi:10.1016/j.jsb.2004.08.002

Labbadia J, Novoselov SS, Bett JS, Weiss A, Paganetti P, Bates GP, Cheetham ME (2012) Suppression of protein aggregation by chaperone modification of high molecular weight complexes. Brain 135(Pt 4):1180-1196. doi:10.1093/brain/aws022

Lawrence A-M, Besir H (2009) Staining of proteins in gels with Coomassie G-250 without organic solvent and acetic acid. J Vis Exp 30:e1350. doi:10.3791/1350

Li J, Qian X, Sha B (2009) Heat shock protein 40: structural studies and their functional implications. Protein Pept Lett 16(6):606-612

Linse S, Cabaleiro-Lago C, Xue WF, Lynch I, Lindman S, Thulin E, Radford SE, Dawson KA (2007) Nucleation of protein fibrillation by nanoparticles. Proc Natl Acad Sci U S A 104(21):8691-8696. doi:10.1073/pnas.0701250104

Lotz GP, Legleiter J, Aron R, Mitchell EJ, Huang SY, Ng C, Glabe C, Thompson LM, Muchowski PJ (2010) Hsp70 And Hsp40 functionally interact with soluble mutant huntingtin oligomers in a classic ATP-dependent reaction cycle. J Biol Chem 285(49):38183-38193. doi:10.1074/jbc.M110.160218

Marsh JL, Walker H, Theisen H, Zhu YZ, Fielder T, Purcell J, Thompson LM (2000) Expanded polyglutamine peptides alone are intrinsically cytotoxic and cause neurodegeneration in Drosophila. Hum Mol Genet 9(1):13-25. doi:10.1093/hmg/9.1.13

Menzies FM, Hourez R, Imarisio S, Raspe M, Sadiq O, Chandraratna D, O'Kane C, Rock KL, Reits E, Goldberg AL, Rubinsztein DC (2010) Puromycin-sensitive aminopeptidase protects against aggregation-prone proteins via autophagy. Hum Mol Genet 19(23):4573-4586. doi: $10.1093 / \mathrm{hmg} / \mathrm{ddq} 385$

Morimoto RI (2008) Proteotoxic stress and inducible chaperone networks in neurodegenerative disease and aging. Genes Dev 22(11):1427-1438. doi:10.1101/gad.1657108

Morley JF, Brignull HR, Weyers JJ, Morimoto RI (2002) The threshold for polyglutamine-expansion protein aggregation and cellular toxicity is dynamic and influenced by aging in Caenorhabditis elegans. Proc Natl Acad Sci U S A 99(16):10417-10422. doi:10. 1073/pnas.152161099

Novak MJ, Tabrizi SJ (2010) Huntington's disease. BMJ 340:c3109

Papaleo E, Invernizzi G (2011) Conformational diseases: structural studies of aggregation of polyglutamine proteins. Curr Comput Aided Drug Des 7(1):23-43

Qian YQ, Patel D, Hartl FU, McColl DJ (1996) Nuclear magnetic resonance solution structure of the human Hsp40 (HDJ-1) Jdomain. J Mol Biol 260(2):224-235. doi:10.1006/jmbi.1996.0394 
Ramazzotti M, Monsellier E, Kamoun C, Degl'Innocenti D, Melki R (2012) Polyglutamine repeats are associated to specific sequence biases that are conserved among eukaryotes. PLoS One 7(2):e30824. doi:10.1371/journal.pone.0030824

Raspe M, Gillis J, Krol H, Krom S, Bosch K, van Veen H, Reits E (2009) Mimicking proteasomal release of polyglutamine peptides initiates aggregation and toxicity. J Cell Sci 122(18):3262-3271. doi: $10.1242 /$ jcs. 045567

Ratovitski T, Gucek M, Jiang H, Chighladze E, Waldron E, D'Ambola J, Hou Z, Liang Y, Poirier MA, Hirschhorn RR, Graham R, Hayden MR, Cole RN, Ross CA (2009) Mutant huntingtin N-terminal fragments of specific size mediate aggregation and toxicity in neuronal cells. J Biol Chem 284(16):10855-10867. doi:10.1074/jbc.M804813200

Ren H, Nagai Y, Tucker T, Strittmatter WJ, Burke JR (2001) Amino acid sequence requirements of peptides that inhibit polyglutamineprotein aggregation and cell death. Biochem Biophys Res Commun 288(3):703-710. doi:10.1006/bbrc.2001.5783

Sarparanta J, Jonson PH, Luque H, Udd B (2011) LGMD1D mutations impair the antiaggregation activity of DNAJB6. Neuromuscul Disord 21(9-10):668. doi:10.1016/j.nmd.2011.06.849

Schaefer MH, Wanker EE, Andrade-Navarro MA (2012) Evolution and function of $\mathrm{CAG} /$ polyglutamine repeats in protein-protein interaction networks. Nucleic Acids Res 40(10):4273-4287. doi:10. 1093/nar/gks011

Stoscheck CM (1990) Quantitation of protein. Methods Enzymol 182:50-68

Streets AM, Sourigues Y, Kopito RR, Melki R, Quake SR (2013) Simultaneous measurement of amyloid fibril formation by dynamic light scattering and fluorescence reveals complex aggregation kinetics. PLoS One 8(1):e54541. doi:10.1371/journal.pone.0054541
Suzuki H, Noguchi S, Arakawa H, Tokida T, Hashimoto M, Satow Y (2010) Peptide-binding sites as revealed by the crystal structures of the human Hsp40 Hdj1 C-terminal domain in complex with the octapeptide from human Hsp70. Biochemistry 49(39):8577-8584. doi:10.1021/bi100876n

Tyedmers J, Mogk A, Bukau B (2010) Cellular strategies for controlling protein aggregation. Nat Rev Mol Cell Biol 11(11):777-788. doi:10.1038/nrm2993

Venkatraman P, Wetzel R, Tanaka M, Nukina N, Goldberg AL (2004) Eukaryotic proteasomes cannot digest polyglutamine sequences and release them during degradation of polyglutamine-containing proteins. Mol Cell 14(1):95-104. doi:10.1016/s1097-2765(04) 00151-0

Wellington CL, Ellerby LM, Hackam AS, Margolis RL, Trifiro MA, Singaraja R, McCutcheon K, Salvesen GS, Propp SS, Bromm M, Rowland KJ, Zhang T, Rasper D, Roy S, Thornberry N, Pinsky L, Kakizuka A, Ross CA, Nicholson DW, Bredesen DE, Hayden MR (1998) Caspase cleavage of gene products associated with triplet expansion disorders generates truncated fragments containing the polyglutamine tract. J Biol Chem 273(15):9158-9167

Wetzel R (2012) Physical chemistry of polyglutamine: intriguing tales of a monotonous sequence. J Mol Biol 421(4-5):466-490. doi:10. 1016/j.jmb.2012.01.030

Wu JW, Breydo L, Isas JM, Lee J, Kuznetsov YG, Langen R, Glabe C (2010) Fibrillar oligomers nucleate the oligomerization of monomeric amyloid beta but do not seed fibril formation. J Biol Chem 285(9):6071-6079. doi:10.1074/jbc.M109.069542

Zoghbi HY, Orr HT (2000) Glutamine repeats and neurodegeneration. Annu Rev Neurosci 23:217-247. doi:10.1146/annurev.neuro.23.1. 217 